\title{
EX ORDIUM
}

\section{LA COMPETENZA INTERCULTURALE IN AMBITO SCOLASTICO: \\ IL RUOLO DEI CONTESTI ORGANIZZATIVI \\ di Marta Milani}

Spesso v'è la tendenza ad agganciare la responsabilità dello sviluppo di competenza interculturale nel contesto scolastico al singolo docente; ma è davvero così? $\mathrm{O}$ gli insegnanti necessitano di essere supportati da una gamma o mescolanza di consapevolezze, strumenti, organi e apparati che costituiscono ingredienti indispensabili al processo? Per offrire reali opportunità di promozione ed esercizio di competenza interculturale è necessario infatti adottare una prospettiva interazionista ed ecologica (Bronfenbrenner, 1986), che ricerchi le condizioni globali dell'aiuto al cambiamento, dal momento che non è possibile - e sufficiente dare una risposta individuale a un problema sistemico.

Il presente articolo focalizza la riflessione sull'urgenza di istituire/attuare servizi, procedure e norme congrui per contribuire a rendere la scuola un universo in cui viene incoraggiato e incentivato il senso di appartenenza, l'empowerment personale e l'esercizio della cittadinanza attiva.

There is often a tendency to assume that the individual teacher is responsible for the development of intercultural competence at school; but is that really true? Or do teachers need to be supported by a range or mix of awareness, tools, bodies and apparatus which are essential elements in the process? In order to offer real opportunities of promotion and exercise of intercultural competence it is indeed necessary to adopt an interactional and ecological perspective (Bronfenbrenner, 1986), that seeks the 
global conditions favoring change as it is neither possible nor sufficient to provide an individual response to a systemic problem.

This article reflects on the urgent need to establish/implement adequate services, procedures and rules to help make school an environment in which the sense of belonging, the personal empowerment and the exercise of active citizenship are encouraged and incentivized.

\section{Introduzione}

Oggigiorno le globalizzazioni (economiche, culturali, politiche e ambientali), le migrazioni, i viaggi, lo sviluppo di comunità online delocalizzate rendono sempre più difficile immaginare le culture come realtà granitiche, stabili e coerenti (India \& Rosaldo, 2002; Portera \& Dusi, 2016). La trama demografica sta cambiando radicalmente la fisionomia mondiale: in soli 20 anni il numero di migranti è raddoppiato, passando dai 33,9 milioni del 1997 ai 65,6 milioni del 2016 (Centro Studi e Ricerche IDOS, 2017) ${ }^{1}$. Per quanto concerne, nello specifico, l'Italia, tra il 2007 e il 2016, nonostante gli strascichi negativi della crisi, gli stranieri sono aumentati di 2.023.317 unità. In particolare, gli arrivi via mare sono passati dai 153.842 nel 2015 a 181.436 nel $2016(+17,9 \%)$ e le richieste d'asilo da 84.085 a 122.960 (+ 46,2\%), facendo registrare i valori più alti degli ultimi anni. Tali dati scquarciano il velo di Maya su alcune questioni: la prima, e più importante, è che il fenomeno migratorio non si manifesta in maniera estemporanea e spuria, ma ha una caratteristica di strutturalità - così come messo in luce da Bauman (2016): «Il diverso è in casa nostra e non se ne andrà» (p.

${ }^{1}$ Appadurai (1991) ha coniato il concetto di "ethnoscapes" per indicare «il panorama di persone che ricompongono il mutevole mondo nel quale viviamo: turisti, immigrati, rifugiati, esiliati, lavoratori stranieri e altri gruppi e persone in movimento [che] costituiscono un tratto distintivo del mondo e sembrano incidere sulle politiche di e tra le nazioni ad un livello finora senza precedenti» (p. 192). 
3) -, con buona pace degli epigoni del negazionismo e razzismo di Stato, amplificati surrettiziamente dai media; la seconda ha a che vedere con la crisi attuale che, secondo Sennett (2008), non è tanto o solo crisi della capacità produttiva, ma soprattutto l'indebolimento e/o abbruttimento della "sociability", ovvero la capacità di tessere forme di relazione stabili, soddisfacenti e di lunga durata. La sociabilità richiede di accettare lo straniero come presenza preziosa per la società ed è l'opposto del tribalismo reazionario ("noi contro loro") da cui l'apertura delle frontiere, in particolare l'accordo di Schengen, è stata accompagnata. Le migrazioni non vengono gestite, ma ostacolate, alimentando le paure, erigendo muri ${ }^{2}$ (congiuntamente a un solido apparato di sicurezza) che, pensati come deterrenti, hanno avuto invece un impatto devastante soprattutto a livello sociale. Per questo il dialogo interculturale trova senso e necessità ancora maggiori in ambito scolastico, da sempre fucina multiculturale e palestra di cittadinanza e mediazione. In tal senso, l'acquisizione, il mantenimento e perfezionamento continuo di competenza interculturale per l'inclusione e l'effettiva partecipazione democratica sono un diktat che molti Istituti dovrebbero abbracciare senza riserve, a prescindere dalla normativa esistente ${ }^{3}$. Ciò significa che lo sviluppo di competenza interculturale non è appannaggio del singolo

2 Basti pensare che, nel 1989, quando fu abbattuto il muro di Berlino, esistevano solo 15 muri di confine. Oggi sono 70, tre quarti dei quali costruiti negli ultimi 25 anni (Jones, 2016).

${ }^{3}$ Il riferimento è - tra le normative storiche che hanno consentito alla pedagogia interculturale di attecchire nel terreno della scuola italiana nell'arco del ventennio 1989-2009 - alla "Via italiana per la scuola interculturale e l'integrazione degli alunni stranieri" (2007), al "Libro bianco sul dialogo interculturale" (2008), così come nella Raccomandazione CE del 18 dicembre 2006, n. 962, dove si legge: «Dato che la globalizzazione continua a porre l'Unione europea di fronte a nuove sfide, ciascun cittadino dovrà disporre di un'ampia gamma di competenze chiave per adattarsi in modo flessibile a un mondo in rapido mutamento e caratterizzato da forte interconnessione. L'istruzione nel suo duplice ruolo - sociale ed economico - è un elemento determinante per assicurare che i cittadini europei acquisiscano le competenze chiave necessarie per adattarsi con flessibilità a siffatti cambiamenti» (p. 13). 
individuo (docente o alunno che sia), ma è una responsabilità che investe l'ente nel suo complesso, attraverso una diversa lettura e gestione dei compiti istituzionali. È d'uopo, pertanto, che la leadership di tali istituzioni in primis si adoperi per costruire un contesto che consenta l'attuazione di norme, procedure, atteggiamenti e stili di lavoro che possano condurre a un servizio interculturalmente competente (Ciancio, 2014). Ciò è possibile e auspicabile anche in virtù della funzione strategica che la scuola dell'autonomia attribuisce a una dirigenza che, però, dovrà essere preparata a gestire sia le risorse interne alla scuola (in termini di organizzazione del personale, dispositivi di accoglienza e di promozione dell'inclusione, laboratori linguistici, procedure amministrative e di valutazione, per citarne alcuni) che quelle esterne, con riferimento ai rapporti con l'amministrazione statale, con gli altri Istituti (continuità verticale) e con la società civile del territorio (continutà orizzontale). Non è necessario che la scuola appronti strutture, procedure o modelli operativi ad hoc per gli immigrati; piuttosto, si deve agire sul sistema nel suo complesso in ottemperanza al paradigma della pedagogia interculturale, per offrire servizi equi e di qualità ad ognuno, a prescindere dall'appartenenza etnica. Si tratta di un lavoro longitudinale - non scevro da ostacoli - che può essere implementato a piccoli passi, ma che deve essere perseguito con tenacia, dal momento che la competenza interculturale, ad oggi, rappresenta una delle strategie principali per garantire l'efficacia e l'efficienza dei servizi pubblici e di una nazione.

\section{La struttura e l'azione della competenza interculturale}

Quando si parla di "competenza interculturale" si intende "[...] the appropriate and effective management of interaction between people who, to some degree or another, represent different or divergent affective, cognitive, and behavioural orientations to the world" (Spitzberg \& Changnon, 2009, p. 7). Tale definizione, nella canea delle cocettualizazioni e prospettive - talora antinomiche - presenti in letteratura, ha il merito di enucleare due aspetti 
di particolare rilevanza: il primo riguarda il fatto che non è possibile distingure nettamente le competenze interculturali dalle competenze tout court: "[...] così come nella società complessa e interdipendente la pedagogia interculturale è da intendere come la migliore risposta educativa per tutti, tutte le competenze interpersonali facilitano le relazioni interculturali" (Portera, 2013a, pp. 148-149); il secondo, invece, fa riferimento alla natura multifattoriale della competenza interculturale e, in particolare, alle tre dimensioni fondamentali attraverso le quali essa si dispiega: l'area del sapere/contenutistica, lo sviluppo di abilità professionali e la maturazione di atteggiamenti personali.

Non può esserci competenza senza un bagaglio strutturato di conoscenze (Cambi, 2005). E ciò significa amore per il sapere, riconoscendone la sua funzione agalmatica. La cultura offre infatti le coordinate per ritarare e calibrare convinzioni e pensieri talvolta granitici, restituendogli il loro peso specifico. Essa consente di avere chiavi di lettura sugli avvenimenti che non siano faziose, mistificanti o frutto di una vorace ma improduttiva dossografia, e di nutrirsi di pensieri lumeggiati interrogando - per dirla con Foucault (1984) - «la verità nei sui effetti di potere e il potere nei suoi discorsi di verità» (p. 40). Ciò non vuol dire essere aporetici per partito preso, ma piuttosto far proprio il monito kantiano "Sapere aude" per affrancarsi dal giogo di idee surrettizie, sovente alimentate dai (corto)circuiti (dis)informativi attuali. Il possesso dei saperi dovrà essere pertanto rigoroso, organico e interdisciplinare, focalizzandosi soprattutto su tematiche umane e sociali (Santerini, 2017): migrazioni e differenze etniche, Paesi di provenienza dei migranti, composizione della società multiculturale, diversità e analogie che intercorrono tra idiomi in termini di comunicazione verbale e non verbale. La creazione e la valorizzazione della competenza non può non poggiare le sue fondamenta su saperi esperti; i quali, per non risultare vacui, dovranno essere sempre connessi con l'area dell'azione e dell'esperienza (il "saper fare"), innescando un circolo virtuoso e fruttifero tale per cui gli eventi alimentano la conoscenza e viceversa. Non è sufficiente, infatti, agire a livello cognitivo per contrastare la proliferazione 
teratomorfa di pensieri e atteggiamenti razzisti: è indispensabile mettersi in gioco in ambiti che incoraggino la capacità di decentramento, la disponibilità, la sensibilità, l'apertura e, soprattutto, l'autoriflessività. Quest'ultima è legata a

un processo interno con cui esaminiamo noi stessi, inclusi i nostri valori, nell'esercizio della coscienza critica [...] un processo che dipende dall'idea di un sé che si trasforma, che emerge e cambia continuamente in quanto interagiamo con gli altri, l'ambiente e la sfera pubblica (Cunliffe, 2002, p. 35).

Ad avere un ruolo preminente è il pensiero critico, il quale consente di fare palinodia di tesi pregresse talora scricchiolanti ed esautorate per leggere e interpretare consapevolmente gli eventi sociali. Ricostruire le esperienze vissute in modo analitico, individuando le azioni compiute, estrapolando le emozioni provate e valutando gli esiti sortiti da comportamenti e atteggiamenti messi in campo, chiama in causa le cosidette funzioni esecutive calde (ovvero l'auto-regolazione cognitiva in situazioni con rilevanza motivazionale-emotiva) congiuntamente alle funzioni esecutive fredde (auto-regolazione cognitiva) (Kerr \& Zelazo, 2004) attraverso un atteggiamento metacognitivo e introspettivo. Quest'ultimo, mediante abilità di pianificazione, automonitoraggio e auto-controllo, consente un accesso consapevole alla conoscenza e al pensiero capace poi di sortire riverberi progettuali sull'agire pratico ${ }^{4}$ (Mezirow, 2003); viene cioè facilitata la capacità di prendere decisioni, definire obiettivi, pianificare gli step necessari e avere dei sistemi di controllo di tipo cognitivo e sociale per capire se si sta procededo bene, affinando in tal guisa quella parte di intelligenza sociale, interpersonale, che influisce poi enorme-

${ }^{4}$ Scrivono a tal proposito Santerini e Reggio (2004): «attraverso la professionalità riflessiva è possibile realizzare la personalizzazione della capacità di affrontare la pluralità e la complessità dei bisogni e di organizzare l'esperienza, questione che risulta strategica sotto il profilo della dimensione interculturale» (p. 261). 
mente sul funzionamento di quella intrapersonale (Gardner, 1993).

Il sinolo del concetto di "competenza interculturale" va pertanto ricercato nell'intreccio di elementi che lo costituiscono e che interagiscono tra di loro in modo fluttuante, non lineare; da qui l'andamento diacronico, la mobilità perpetua, la natura intrinsecamente asintotica. Vi è un nocciolo inscindibile di pensiero e azione, di significazione personale e intersoggettiva che si sviluppa in situazione attraverso un processo costante di tipo proattivo e retroattivo, che ne qualifica la dimensione progettuale. Per siffatta ragione, nell'iter di sviluppo della competenza, non vi è mai un vero punto di arrivo (Deardorff, 2009; Milani, 2017; Portera, 2013a). Il costrutto di competenza interculturale collima perciò con approcci che lo vorrebbero incasellato entro un framework di standardizzazione - di matrice taylorista e, successivamente, behavorista-comportamentista - dacché richiede quella capacità di rieditare, ricreare e combinare in maniera sempre nuova e creativa conoscenze, comportamenti e atteggiamenti. Per saper usare in maniera flessibile una capacità/abilità infatti, non solo bisogna acquisirla, ma anche inibire l'uso di una precedente, meno valida, tenendo a bada le risposte automatiche o prepotenti (controllo inibitorio); in tal senso, è cruciale saper accedere all'informazione immagazzinata nella memoria a lungo termine e tenere presente ora questa ora quella nella memoria di lavoro per monitorare le operazioni cognitive e socio-emotive in corso (Dunlosky, Bjork \& Graesser, 2008). Sono le cosidette "competenze fantacognitive" (Frabboni, 2000), il cui nerbo è costituito da un adattamento flessibile teso ad affrontare problemi inediti non riconducibili a singole prestazioni.

La competenza realmente generativa e proattiva, oltre a essere caratterizzata da responsabilità, autonomia e libera scelta, deve poter accedere a tutte quelle risorse che consentono di realizzare una progettualità di qualche tipo. Ecco perché lo sviluppo di competenza deve essere sempre sostenuto da processi di approfondimento e acquisizione progressiva così come di ampliamento/ridefizinizione dei contesti di esercizio. 


\section{Elementi contestuali che influiscono sullo sviluppo di competenza interculturale}

La conditio sine qua non affinché un percorso di educazioneformazione alla competenza interculturale per studenti e studentesse abbia successo è, fuor di dubbio, l'acquisizione della stessa in primis da parte del personale docente. Tuttavia, non è pensabile/auspicabile che tale categoria paidetica sia loro esclusivo appannaggio; il lavoro per la costruzione di un autentico clima di inclusione e integrazione positiva richiede, infatti, la collaborazione e la responsabilità di tutti gli attori, organi e apparati (statali e non) che gravitano intorno alla galassia scuola. Infatti, come emerso da una precedente ricerca (Milani, 2015), anche la dimensione contestuale-organizzativa e la relativa presenza/assenza di risorse, supporti e strutture (in termini di pianificazione curricolare, scelta dei materiali e dei metodi didattici, procedure e normative scolastiche, ma anche le scelte di dettaglio) influiscono sullo sviluppo di competenza interculturale. Per raggiungere risultati di spessore e non transeunti, ma anche per far sì che qualsiasi dichiarazione politica ufficiale in materia non resti lettera morta, è necessario appellarsi a una rinnovata governance scolastica che si basi su un lavoro d'équipe e su un'intenzionalità comune capace di rendere più efficace l'impegno profuso. A tal proposito, la figura dirigenziale riveste un ruolo di primaria importanza, a condizione che qualunque decisione organizzativa venga accompagnata da un congruo stanziamento di risorse umane e strutturali con le quali rendere effettivo l'impegno nonché valutare il lavoro svolto. E quindi molto importante tenere a mente che

senza il concreto e reale supporto, seppur minimo, delle principali figure manageriali dell'amministrazione, gli sforzi prodotti dai singoli individui o da piccoli gruppi dello staff non sarebbero probabilmente in grado di creare e successivamente sostenere complessi cambiamenti strutturali [...] (Ciancio, 2014, p. 40).

L'iter che conduce al raggiungimento della competenza interculturale all'interno del sistema scolastico richiede strategie duttili e multifattoriali in cui partecipazione, formazione e collaborazio- 
ne divengono i punti di Archimede su cui fare perno. Nello specifico, si tratta di attivare a livello sistemico e organizzativo dei processi chiave quali:

- mission, vision, politiche e linee guida che sposino appieno la causa del raggiungimento di competenza interculturale: la scuola, così come ogni azienda, gode di un'autonomia ${ }^{5}$ ammnistrativa, gestionale, didattica e organizzativa nell'antro delle normative e delle regole emanate dallo Stato. Ciò consente di poter dare risposte mirate alle inedite esigenze che, soprattutto oggigiorno, in una società sempre più proteiforme, impattano sulla scuola, e di rimuovere al contempo gli ostacoli istituzionali e/o eventuali ostruzionismi che potrebbero frapporsi alla realizzazione dell'obiettivo finale. Solo adottando un approccio flessibile sarà possibile operare e incidere non solo a livello dei gruppi già deputati a lavorare su tematiche interculturali (funzione strumentale e/o referenti, commissione intercultura o integrazione), ma anche a livello personale, dei singoli docenti, dacché motivazione, impegno e spinta a divenire competenti sono direttamente proporzionali agli sforzi compiuti in tal senso dalle componenti organizzative dell'ente. Infatti, laddove si frappongano resistenze organizzative e strutturali, non solo sarà difficile raggiungere un livello minimo di competenza interculturale, ma si innescherà una forma di abulia demotivante per cui risulterebbe complesso mantenere il grado precedentemente raggiunto;

- forza lavoro variegata, competente e qualificata: tale pilastro si riferisce alla necessità di reperire risorse umane, al mantenere piani di assegnazione culturalmente appropriati e/o di mediazione interculturale e servizi di interpretariato senza dimenti-

${ }^{5}$ L'autonomia degli Istituti è stata introdotta dalla legge n. 59/1997 (la cosidetta "riforma Bassanini") e realizzata successivamente dal DPR 275/1999; essa è lo strumento attraverso cui l'istituzione scolastica può adottare metodi di lavoro, tempi di insegnamento, soluzioni funzionali alla realizzazione dei piani dell'offerta formativa e alle esigenze e vocazioni di ciascun alunno. 
care, però, l'acquisizione di competenze linguistiche personali esponenzializzando il numero di docenti, dirigenti, personale ata (almeno) bilingue. Rispetto a quest'ultimo punto, sarebbe buono stilare una sorta di "anagrafe" del personale, per meglio capitalizzare il valore aggiunto - linguistico/culturale - di ogni persona;

- impegno nella comunità e partnership: i singoli professionisti che lavorano all'interno di un servizio pubblico quale è la scuola non sono monadi isolate, ma fanno parte di un sistema aperto. È importante, perciò, sia sviluppare reti con gruppi delle comunità etniche per favorime il diretto coinvolgimento, sia attivare partership con altre organizzazioni - statali e non - , il welfare e i servizi tutti per rendere le politiche e i processi più congruenti;

- pubblicazione e divulgazione delle informazioni chiare, disambiguate, pertinenti e in una lingua e forma che sia congruente alla capacità di comprensione in prims delle famiglie. Avere a disposizione degli strumenti multilingue può contribuire a rendere comprensibili azioni, procedure, protocolli, modulistiche e un consenso informato chiaro e trasparente. Utili sarebbero anche $\mathrm{i}$ totem informativi/illustrativi multilingue perché, sovente, i cittadini stranieri dimostrano di avere lacune anche nella lingua ufficiale del proprio Paese. Tutto ciò costituisce un primo fondamentale passo nel processo di attivazione di empowerment delle comunità immigrate, perché se il gruppo etnico specifico o l'individuo coinvolto non hanno pieno accesso alle informazioni trasmesse o non comprendono, non si può parlare di integrazione positiva o interazionista (Portera, 2006);

- politica di sostegno: la messa in pratica dei progetti e dei piani relativi allo sviluppo di competenza interculturale da parte della scuola è influenzata anche dal fatto che questa opera all'interno di un sistema politico-culturale estremamente complesso e aperto a tutte le pressioni che possono essere innescate dalla molteplicità di stakeholders individuali e istituzionali. Ecco perché è particolarmente importante impegnarsi 
per garantire equità (Tarozzi, 2015), perché la vera inclusione passa dalla piena accessibilità e fruibilità dei servizi. Se v’è un vuoto istituzionale o sociale di qualche tipo (clima politico sul territorio che non favorisce un programma di lavoro specifico all'interno della scuola, mancate autorizzazioni, ecc.), difficilmente può esserci pieno sviluppo di competenza interculturale; infatti, anche quando un solo elemento del sistema è claudicante o deficitario, tale mancanza si ripercuote negativamente su altre fasi del processo;

- riconoscimento e valorizzazione della diversità culturale attraverso modalità e percorsi che consentano di garantire momenti per l'ascolto e autentica comunicazione interpersonale ${ }^{6}$. Fare attenzione alla forma in cui è trasmesso e ricevuto un messaggio perché potrebbe essere ricevuto e/o interpretato dal ricevente in una modalità culturale tale da apparirgli/le artefatta, offensiva, irrilevante...;

- condurre/stimolare e incoraggiare una strategia di valutazione organizzativa, ossia un'autovalutazione interna e costante della competenza interculturale per valutare sia fino a che punto la scuola sta rispondendo agli specifici bisogni linguisticoculturali dei diversi gruppi etnici che ne costituiscono la variegata geografia sia i punti di forza e debolezza al fine di sviluppare successivamente piani strategici/linee guida nonché meccanismi di controllo che inglobino sistematicamente politiche, modalità d'azione, assetti e metodi operativi. Tale presa di consapevolezza a partire da congetture e convinzioni rappresenta il viatico per identificare in un secondo momento azioni di formazione/educazione continua con lo scopo di ridurre eventuali barriere e/o criticità. In tal senso, workshop, singoli

${ }^{6}$ Scrive La Marca (2015): «La comunicazione interpersonale che richiama quella interculturale si esplica tramite tre direttrici: la prima è la traiettoria di senso concessa dall'ascolto, la seconda è quella determinata dal dialogo, la terza è quella data dall'empatia. Ascolto, dialogo ed empatia sono tre dimensioni della comunicazone come comprensione dell'altro e del proprio sé che aiutano e sostengono il soggetto adulto nel difficile rapport con il mondo» (p. 144). 
corsi brevi, moduli, seminari di varia durata, corsi integrati potrebbero rappresentare una soluzione ottimale; l'importante è che si tratti di metodi partecipativi, esperienziali e interdisciplinari $^{7}$ e che arrivino ad agire su quattro livelli: personale, professionale, organizzativo e sistemico;

- acquisizione e istituzionalizzazione della conoscenza culturale: sarà importante esaminare la particolare storia e organizzazione dei sistemi delle credenze e dei valori culturali, degli usi e costumi, delle dinamiche intra e inter culturali delle famiglie immigrate di ciascuna delle comunità presenti nel territorio. Particolare attenzione, poi, dovrà essere posta al contesto sociale in cui è inserita la famiglia immigrata - poiché potrebbe essere estremamente disagiato - così come osservare l'interazione tra i gruppi di minoranze etniche e le loro istituzioni sociali e se sussistano prove di eccessive pressioni esercitate su tali minoranze. Non meno importanti nella raccolta dati sono le informazioni inerenti la presenza di lamentele, gli incidenti critici e, in generale, la mancanza di soddisfazione di tutti gli attori coinvolti nel circuito scolastico. Il desiderio, la volontà e la passione di entrare in sintonia con l'Altro diverso da sé, di conoscere, sostenere e incoraggiare va pertanto individuato, canalizzato e coltivato da parte di entrambi gli interlocutori: scuola e utenza, in particolare quella di origine straniera. Infatti, lo sforzo al raggiungimento di competenza interculturale deve essere sempre bidirezionale e richiede l'impegno di tutti: popolazione autoctona e immigrati.

${ }^{7}$ Quali ad esempio: role play, problem solving, problem based learning, action learning, metodi narrativi (storytelling, letteratura della migrazione, metodi autobiografici, produzione audiovisiva, laboratori di scrittura, narrazioni collettive, diario o scrapbook fotografico, counter-narrative, ecc.), metodi espressivi (disegno e pittura, metodi a mediazione corporea, composizioni manuali simboliche, laboratori sulle emozioni, arte cooperativa, musica e danza, teatro, laboratori fotografici, interpretazioni di immagini, ecc.), dibattito bidirezionale su questioni specifiche, reflective practice. 


\section{Annotazioni conclusive}

Oggigiorno, in una società sempre più complessa e diversificata, lo sviluppo della competenza interculturale dovrà avvenire secondo un modello interazionista ed ecologico (Bronfenbrenner, 1986) per giungere alla school readiness; ovvero non si tratta più - o meglio: non solo - di rilevare la competenza dello studente, ma anche quella della famiglia, dei servizi offerti, della comunità nel suo complesso. Ciò significa che, nell'attuale scuola multiculturale, per raggiungere un efficiente livello di competenza, inclusione, qualità ed equità, vi sono almeno quattro componenti sulle quali è necessario investire: $i$ singoli individui, le formazioni gruppali che operano nell'organizzazione, le sub-organizzazioni afferenti al sistema e il sistema nel suo complesso. Tali elementi dovranno operare in modo sinergico, omnicomprensivo e flessibile (Ciancio, 2014); omnicomprensivo in quanto un sistema è, di fatto, la somma del personale che vi lavora, ma, al contempo, duttile perché, contrariamente ad alcuni approcci allo sviluppo di competenza interculturale presenti in letteratura - il modello di Bennett ${ }^{8}$ (1993) in primo luogo -, fa riferimento a una dimensione processuale in cui le varie fasi non sono necessariamente conseguenti l'una all'altra in uno specifico ordine. Infatti, talvolta può accadere che alcuni elementi assumano in via temporanea un "ruolo guida" nell'iter, per poi magari perdere di rilevanza o anche cessare e infine riprendere nuovamente importanza quando le circostanze lo richiedono.

La natura cangiante intrinseca alla competenza interculturale obbliga cioè a procedere avanti e indietro lungo il percorso, in base ai momenti, alle specifiche circostanze nonché alle barriere

8 Il Development Model of Intercultural Sensitivity (DIMS) di Bennett (1993) è stato realizzato per dimostrare in che modo le persone sviluppino la percezione della diversità culturale e come, con l'aumentare della complessità e del numero di esperienze di contatti, aumenti anche la competenza interculturale dell'individuo. Sei gli stadi previsti, suddivisi in due fasi che si snodano da un orientamento etnocentrico a uno etnorelativo: denial, defense, minimization, acceptance, adaptation e integration. 
presenti (Deardorff, 2009; Portera, 2013b). Quest'ultime possono essere di varia natura: sistemiche, organizzative, professionali e personali. Rispetto alle prime, si tratta della mancanza di mediatori culturali o servizi di interpretariato, della penuria o totale assenza di materiale negli idiomi maggiormente rappresentativi della comunità locale, così come la difficoltà di leggere la realtà e il contesto locale che, giocoforza, vanno a impattare negativamente sia sul personale che sugli utenti. Per converso, se si desidera fornire un servizio competente ed equo alla variegata popolazione che anima l'universo scuola, è necessario monitorare con constanza il livello di soddisfazione degli utenti immigrati, altrimenti si rimane arroccati a mere congetture e/o dati omeletici. Un altro ostacolo è da ricercarsi nel fatto che spesso è difficile avere persone di origine straniera nei Comitati Genitori e ancora di più lo è ritrovarle investite della carica di rappresentanti di classe'. Ciò, di fatto, come osserva Silva (2008), impedisce di raccogliere informazioni inerenti i bisogni specifici delle famiglie, che finiscono per rimanere taciti; frattanto, la scuola continua a proporre modelli comunicativi e relazionali e/o forgiare iniziative sostanzialmente pensati per un'utenza monoculturale o monolingue. Rispetto a quest'ultimo punto, poi, così come messo in luce da una precedente ricerca (Milani, 2015), è fondamentale rammentare come, in generale, la raccolta dei dati rivesta un'importanza particolare, specie quando è in gioco la piena integrazione degli alunni:

Sembra manchino elementi utili per conoscere in maniera più approfondita gli alunni immigrati e il loro percorso formativo (ad esempio la conoscenza dei sistemi di scolarizzazione dei Paesi di provenienza) e di vita: esistono strumenti a livello di Istituto che consentano ciò? Se i

${ }^{9}$ Lo spettro delle motivazioni di tale assenteismo è vario e racchiude molteplici variabili; tra le principali: impegni lavorativi che cozzano con la pianificazione temporale dei Consigli di classe o d'interclasse, motivi religiosi, distanza culturale dall'approccio con l'istituzione scolastica e valutazione che gli stessi genitori fanno della propria competenza comunicativa in italiano (e quindi dalla difficoltà che ne deriva dal partecipare a incontri e riunioni in cui farebbero fatica a intervenire). 
dati vengono raccolti, sono utilizzati per una migliore integrazione degli alunni? [...]. Solo a partire dalla conoscenza di questa realtà e dall'assunzione di atteggiamenti di sincera enteropatia si potranno comprendere drammi, rischi, pericoli, ma anche potezialità e positività dei vari percorsi e storie che compongono la "classe-mosaico" (Milani, 2015, p. 117).

Le barriere di tipo organizzativo fanno invece riferimento a quegli ostacoli che si frappongono al reclutamento delle diversità, incluso l'intreccio di alleanze con i leader delle differenti comunità presenti sul territorio locale. Il fine ultimo dovrà essere quello della capitalizzazione del know-how, delle risorse umane, del valore aggiunto rappresentato dalle singole biografie esperienziali, professionali ed esistenziali affinché diventino bumus per la proliferazione di creatività, innovazione e progresso. La mancanza di un substrato diverso da quello autoctono in termini di leadership o corresponsabilità gestionale impedisce cioè la germinazione di idee originali - frutto di sguardi e punti di vista inediti - favorendo, al contrario, la stagnazione su strade già battute. Il docente, il dirigente, così come il collaboratore scolastico straniero può rappresentare un apripista per tematiche e/o problematiche che necessitano di riflessioni e coinvolgono persone o situazioni che poco o nulla hanno in comune con la popolazione autoctona.

Vi sono poi le barriere di carattere professionale che, giocoforza, vanno a intersecarsi con quelle personali, dacché i professionisti sono persone in carne e ossa. L'espressione concreta di una competenza interculturale si trova infatti al punto di intersezione nel quale si combinano, oltre alle dimensioni della formazione/educazione formale, della pratica e del contesto d'azione, anche quella legata alla biografia professionale ed esistenziale. L'esperienza e la letteratura (Deardorff, 2009; Portera, 2016), invero, evidenziano che, quando si registrano fenomeni di razzismo, etnocentrismo o vi sono ostacoli di carattere comunicativolinguistico, bias, lacune conoscitive o aspettative differenti, si assiste a una decrescita (o stagnazione) della competenza stessa.

L'educazione permanente, nelle sue svariate articolazioni, gioca pertanto un ruolo chiave giacché non si può divenire compe- 
tenti dal punto di vista interculturale dopo un pacchetto formativo di otto o più ore; la scuola - così come qualsiasi altro servizio - è infatti un sistema aperto posto all'interno di una cornice sociale più ampia in costante evoluzione. Ciò significa altresì che non può esservi un idealtipo morfologico o una strategia didattica blueprint adattabile a ogni situazione; anche se gli studi in materia (Damini, 2011; Lamberti, 2006; Malusà \& Tarozzi, 2017) confermano che l'utilizzo di modalità interattive e coinvolgenti (Cooperative Learning, simulazioni, Action Learning, Reflective Practice, ecc.) possono promuovere fattivamente una cultura di inclusione e integrazione positiva.

\section{Bibliografia}

Appadurai A. (1991). Global Ethnoscapes: Notes and Queries for a Transnational Anthropology. In R.G. Fox (Ed.), Recapturing Anthropology (pp. 191-210). Santa Fe, NM: School of American Research Press.

Bauman Z. (2016). Stranieri alle porte. Roma-Bari: Laterza.

Bennett M.J. (1993). Towards ethnorelativism: A developmental model of intercultural sensitivity. In R.M. Paige (Ed.), Education for the intercultural experience (pp. 109-135). Yarmouth, ME: Intercultural Press.

Bertoni E., \& Rodano, G. (2000) (a cura di). Dossier degli Annali della

Pubblica Istruqione - Il laboratorio della Riforma. Autonomia, Competenze e Curricoli. Firenze: Le Monnier.

Bronfenbrenner U. (1986). Die Ökologie der menscblichen Entwicklung. Stuttgart: Klett.

Cambi F. (2005). Le intenzioni nel processo formativo. Intinerari, modelli, problemi. Pisa: Edizioni Del Cerro.

Centro Studi e Ricerche IDOS (2017). Dossier statistico immigrazione. Roma.

Cesareo V. (2004) (a cura di). L'altro. Identità, dialogo e conflitto nella società plurale. Milano: Vita e Pensiero.

Ciancio B. (2014). Sviluppare la competenza interculturale. Il valore della diversità nell'Italia multietnica. Un modello operativo. Milano: FrancoAngeli.

Commissione Europea (2008). Libro Bianco sul dialogo interculturale. Vivere insieme con pari dignità.

Cunliffe A.L. (2012). Reflexive Dialogical Practice in Management 
Learning. Management Learning, XXXIII, 1, 35-61.

Damini M. (2011). Costruire competenze interculturali attraverso il Cooperative Learning: un percorso di ricerca-azione nella scuola secondaria di secondo grado. Giornale Italiano della Ricerca Educativa, IV , 23-37.

Deardorff D.K. (2009) (Ed.). The sage handbook of intercultural competence. Thousand Oaks: Sage.

Deardorff D.K. (2009). Synthesizing Conceptualizations of Intercultural Competence. In D.K. Deardorff (Ed.), The sage handbook of intercultural competence (pp. 264-269). Thousand Oaks: Sage.

Dunlosky J., Bjork R., \& Graesser A.C. (2008) (Eds.). Handbook of Metacognition and Education. New York: Routledge.

Foucault M. (1984). What is Enlightenment?. In P. Rabinow (Ed.), The Foucault Reader (pp. 32-50). New York: Pantheon Books.

Fox R.G. (1991) (Ed.). Recapturing Anthropology. Santa Fe, NM: School of American Research Press.

Frabboni F. (2000). Disciplinarietà e trasversalità: sapere, comprendere, inventare. In E. Bertoni \& G. Rodano (a cura di). Dossier degli Annali della Pubblica Istruzione - Il laboratorio della Riforma. Autonomia, Competenze e Curricoli (pp. 37-50). Firenze: Le Monnier.

Gardner H. (1993). Multiple Intelligences. New York: Basic Books.

India J.X., \& Rosaldo R. (2002) (Eds.). Anthropology of Globalization: A Reader. Oxford, UK: Blackwell Publishing.

Jones R. (2016). Violent Borders: Refugees and the Right to Move. New York: Verso.

Kerr A., \& Zelazo P.D. (2004). Development of "hot" executive function: the Children Gambling Task. Brain and cognition, 55, 148-157.

La Marca A. (2015). Temi emergenti e aspetti didattici della pedagogia interculturale. In A. Portera, A. La Marca \& M. Catarci (a cura di), Pedagogia interculturale (pp. 143-171). Brescia: La Scuola.

Lamberti S. (2006). Cooperative Learning: una metodologia per la gestione efficace dei conflitti. Padova: Cedam.

Malusà G., \& Tarozzi M. (2017). Ensuring Quality and Equity in an Italian Multicultural Primary School. In A. Portera \& C.A. Grant (Eds.), Intercultural Education and Competences. Challenges and Answers for the Global World (pp. 221-238). Newcastle upon Tyne: Cambridge Scholars Publishing.

Mezirow J. (2003). Apprendimento e trasformazione. Il significato dell'esperienza e il valore della riflessione nell'apprendimento degli adulti. Milano: Raffaello Cortina.

Milani M. (2015). Competenze interculturali a scuola. Verona: QuiEdit.

Milani M. (2017). A scuola di competenze interculturali. Metodi e pratiche peda- 
gogiche per l'inclusione scolastica. Milano: FrancoAngeli.

Osservatorio nazionale per l'integrazione degli alunni stranieri e per l'educazione interculturale (2007). La via italiana per la scuola iterculturale e l'integrazione degli alunni stranieri.

Paige R.M. (1993) (Ed.). Education for the intercultural experience. Yarmouth, ME: Intercultural Press.

Portera A. (2013a). Manuale di pedagogia interculturale. Bari: Laterza.

Portera A. (2013b). Competenze interculturali per la società complessa. In A. Portera (a cura di). Competenze interculturali. Teoria e pratica nei settori scolastico-educativo, giuridico, aziendale, sanitario e della mediazione culturale (pp. 140-152). Milano: Franco Angeli.

Portera A. (2013b) (Ed.). Competenze interculturali. Teoria e pratica nei settori scolastico-educativo, giuridico, aziendale, sanitario e della mediazione culturale. Milano: FrancoAngeli.

Portera A., \& Dusi P. (2016) (a cura di). Neoliberalismo, educazione e competenze intercultutrali. Milano: FrancoAngeli.

Portera A., \& Grant C.A. (2017) (Eds.). Intercultural Education and Competences. Challenges and Answers for the Global World. Newcastle upon Tyne: Cambridge Scholars Publishing.

Portera A., La Marca A., \& Catarci M. (2015) (a cura di). Pedagogia interculturale. Brescia: Editrice La Scuola.

Rabinow P. (1984) (Ed.). The Foucault Reader. New York: Pantheon Books.

Raccomandazione CE del 18 dicembre 2006, n. 962.

Santerini M. (2017). Da stranieri a cittadini. Educazione interculturale e mondo globale. Milano: Mondadori.

Santerini M., \& Reggio P. (2004). La scuola dell'altro. Intercultura e formazione degli insegnanti. In V. Cesareo (a cura di), L'altro. Identità, dialogo e conflitto nella società plurale (pp. 261-302). Milano: Vita e Pensiero.

Sennett R. (2008). L'uomo artigiano. Milano: Feltrinelli.

Silva C. (2008). La relazione tra genitori immigrati e insegnanti nella scuola dell'infanzia. Rivista Italiana di Educazione Familiare, 2, 23-36.

Spitzberg B.H., \& Changnon G. (2009). Conceptualizing Intercultural Competence. In D.K. Deardorff (Ed.), The sage handbook of intercultural competence (pp. 2-52). Thousand Oaks: Sage.

Tarozzi M. (2015) (a cura di). Dallintercultura alla giustizia sociale. Per un progetto pedagogico e politico di cittadinanza globale. Milano: FrancoAngeli. 